\title{
Relationship Between Gender, Age, BMI and Side of Body on the Size and Position of Nerves of the Brachial Plexus in Axilla: Pilot Study
}

\author{
James Lalloo' \\ Pawan Gupta ${ }^{2}$ \\ 'Department of Anaesthesia, Airedale \\ NHS Foundation Trust, Steeton, West \\ Yorkshire, UK; ${ }^{2}$ Department of \\ Anaesthesia, Leeds Teaching Hospitals \\ Trust, Leeds, West Yorkshire, UK
}

Background and Objectives: Studies demonstrate variations in the size and position of the nerves in the brachial plexus. The objective of this pilot study was to determine the effect of age, gender, BMI and side of body on the size and position of these nerves and to determine the feasibility of a further study.

Methods: Twenty healthy volunteers were recruited. The ultrasound position of the nerves was confirmed by a dynamic scan. The size of the nerves was calculated using the freehand calliper tool. A graph was designed to study the position of the nerves. ImageJ was used to analyse the position of the nerves. Student's t-tests were carried out to compare the gender and side of arm with the size of the nerve. Pearson's correlation coefficients were calculated to determine the correlation between BMI and age with the size of the nerves. The position of the nerves was compared between male and female, and left and right sides of the body.

Results: The mean size of the median nerve, musculocutaneous nerve, radial nerve and ulnar nerve was $0.099,0.032,0.179$ and $0.076 \mathrm{~cm} 2$ (males) and $0.091,0.022,0.128$ and $0.026 \mathrm{~cm} 2$ (females), respectively. There were significant differences between the size of nerves and gender in the musculocutaneous, radial and ulnar nerves $(\mathrm{P}<0.05)$. The correlations between the sizes of the nerves with BMI and age were not significant. The position of the radial nerves was found to be variable within the same genders and between males and females. The position of the nerves was variable between the left and right hand side.

Conclusion: The position and size of brachial plexus branches in axilla is very variable. This pilot study highlights the need for further research with larger sample sizes to fully understand the extent and implication of this variability.

Keywords: brachial plexus, ultrasound, anatomy, regional anaesthesia

\section{Introduction}

The brachial plexus provides both the motor and sensory innervation to the upper limb of the body from nerve roots C5-T1. ${ }^{1,2}$ Brachial plexus block has an important role in surgery involving the shoulders, arm, forearm and hands. ${ }^{1,3}$ Axillary brachial plexus block can be used as a sole anaesthetic for upper limb procedures, avoiding the use of general anaesthesia, resulting in fewer complications especially in ASA grade 3 or 4 patients. ${ }^{4,5}$ A number of studies have been conducted into the anatomy of the brachial plexus and how it varies between patients. ${ }^{6-8}$ Despite these studies, there has been little research into how certain factors such as gender, side and body mass index affect the position of various nerves arising from the brachial plexus. 9 These factors may have an impact upon the size and the position of the nerves. This
Correspondence: James Lalloo

Email Jlalloo@doctors.org.uk 
study investigates whether these factors have an impact upon the nerves of the brachial plexus.

Primary Objective: To determine the effect of age, gender, side of body and BMI on the size and position of the nerves of the brachial plexus.

Secondary Outcome: To undertake a pilot study to determine the feasibility of a larger study into the effects of the above factors on the anatomy of the brachial plexus.

\section{Methods}

Twenty volunteers, ten males and ten females, were recruited from the staff of the Hand Unit at Leeds General Infirmary. Twenty volunteers were chosen initially in order to form the basis of a pilot study which could direct further studies on this topic (Table 1). Informed consent was obtained for ultrasound examination of their brachial plexus. Each participant had an ultrasound scan conducted of their axilla at the level of insertion of pectoralis major muscle, and the four major nerves of the brachial plexus (musculocutaneous, median, radial and ulnar) and the axillary artery were identified. The demographics of each participant were recorded. Ethical approval was obtained from the University of Leeds and Health and Research Authority, United Kingdom.

The ultrasound position of the nerves was confirmed by a dynamic scan by two experienced anaesthetic consultants. An USG Sonosite S nerve ${ }^{\mathrm{TM}}$ machine with a 6-13 $\mathrm{MHz}$ probe was used to scan the axillary area. The ulnar nerve was followed up to the ulnar groove, the median nerve was followed up to the elbow along with the brachial artery, the radial nerve was followed until it was seen going around the humeral groove to the lateral side of the arm and the musculocutaneous nerve was followed until it started to branch out in mid upper arm. The images were marked using the inbuilt caliper tool at the time of scanning and confirmed by both consultants. The size of the nerve (cross sectional area in $\mathrm{cm}^{2}$ ) was calculated directly by the machine. The position of the nerves was analysed using Image J@.$^{10} \mathrm{~A}$ graph was designed to demonstrate the location of the nerves. Keeping the axillary artery in the centre, concentric circles were drawn at $1 \mathrm{~cm}$ and these were divided into four quadrants using two perpendicular vertical lines. The centre of each nerve was marked and the co-ordinates at the centre of each of nerves were recorded and plotted onto the graph (Figure 1). This allowed us to mark the precise location of each nerve in relation to the axillary artery.

Once the data had been fully collected, it was analysed using Microsoft Excel ${ }^{\mathrm{TM}}$ 2010. T-tests were carried out in order to compare the size of the nerve between genders (unpaired $t$ test) and the side of arm (paired $t$ test). Pearson's correlation coefficients (PCC) were calculated to determine the correlation between BMI and the size of the nerves. The position of the nerves was visually compared between male/female, and left/right sides of the body.

\section{Results}

\section{Size (Cross Sectional Area) of Nerve}

Table 2 shows the results for both right and left hand side of the body and males and females for each of the four nerves of the brachial plexus. The radial nerve was the largest of the four nerves. There were significant differences in the size of nerves between genders for the musculocutaneous, radial and ulnar nerves ( $\mathrm{P}$ value $<0.05$ ). There was no significant difference between the size of the nerves on the left and right hand side of the body.

\section{Relationship of Nerve Size to BMI and Age}

Table 3 demonstrates the correlation between the size of each of the nerves in the axilla and the BMI and age. The results demonstrate that there are weak positive correlations between the size of the median nerve, radial nerve, ulnar nerve with BMI. In addition, there is a weak positive correlation between the size of the radial nerve and age.

Table I Demographics Data of the Sample Set

\begin{tabular}{|l|c|c|c|c|c|c|c|c|}
\hline Gender & \multicolumn{4}{|c|}{ Male } & \multicolumn{3}{c|}{ Female } \\
\hline BMI & $20-25$ & $25-30$ & $30-35$ & $35-40$ & $20-25$ & $25-30$ & $30-35$ & $35-40$ \\
N & $\mathrm{I}$ & 6 & 2 & 1 & 7 & 2 & 1 \\
\hline Age & $20-29$ & $30-39$ & $40-49$ & $50-60$ & $20-29$ & $30-39$ & $40-49$ & $50-59$ \\
N & 2 & 3 & 3 & 2 & 5 & 2 & 2 \\
\hline
\end{tabular}

Abbreviations: BMI, body mass index kg/m2; N, number; Age, in years. 

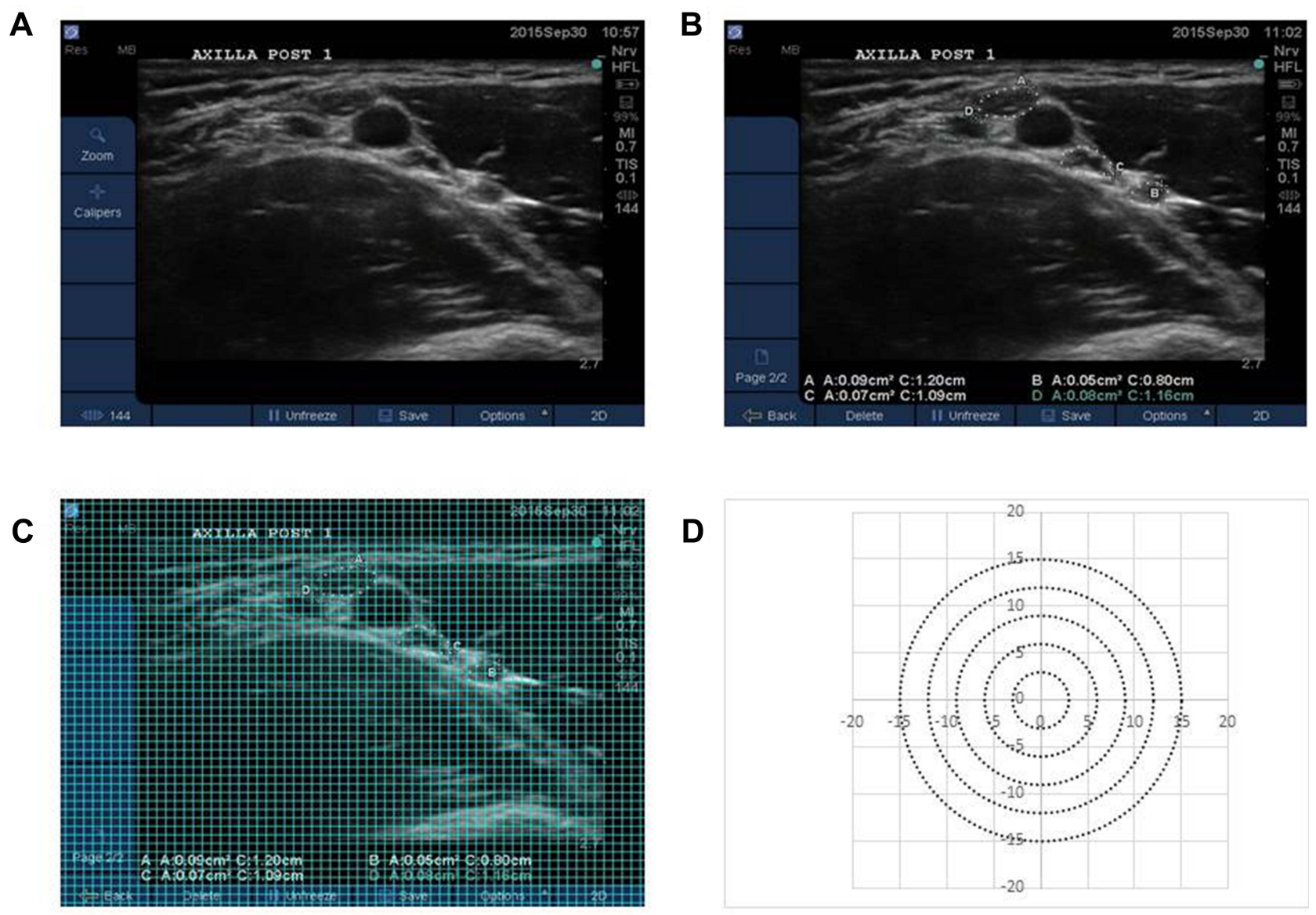

Figure I (A) Original ultrasound image of brachial plexus. (B) Freehand selection of nerves . (C) Image image with overlaying grid. (D) Graph used to compare the position of the nerves of the brachial plexus.

\section{Position of Nerves}

Figure 2 shows the distribution of the nerves of the brachial plexus in males and females. The most striking finding is the general distinct variation in the position of nerves in subjects of the same gender. The figure demonstrates that for the median nerve, the musculocutaneous nerve and the radial nerve the most common position of the nerve varied between males and females.

Figure 3 demonstrates the distribution of the nerves on the right and left-hand sides of the body. It shows that for the radial nerve the most common position of the nerve varies between right and left. However, the most marked observation is the distinct variation in the position of the nerves between individuals on both sides of the body.

\section{Discussion}

Analysing the position of the nerves in this study demonstrated that the position of each nerve varied greatly between each participant. There was variation visualized between the most common position of the median,

Table 2 Mean and Standard Deviation (SD) for the Right and Left Hand Side and Males and Females for the Four Nerves of the Brachial Plexus

\begin{tabular}{|c|c|c|c|c|c|c|c|c|c|c|c|c|}
\hline \multirow[t]{2}{*}{ Variable } & \multicolumn{3}{|c|}{ Median (Units $\mathrm{cm}^{2}$ ) } & \multicolumn{3}{|c|}{ Musculocutaneous } & \multicolumn{3}{|c|}{ Radial } & \multicolumn{3}{|c|}{ Ulnar } \\
\hline & Mean & SD & $P$ value & Mean & SD & P value & Mean & SD & $P$ value & Mean & SD & $P$ value \\
\hline Male & 0.099 & 0.019 & 0.1882 & 0.032 & 0.014 & 0.0068 & 0.179 & 0.048 & 0.0002 & 0.076 & 0.012 & 0.0004 \\
\hline Female & 0.091 & 0.036 & & 0.022 & 0.008 & & 0.128 & 0.033 & & 0.062 & 0.013 & \\
\hline Right & 0.092 & 0.021 & 0.2446 & 0.028 & 0.01 & 0.4047 & 0.152 & 0.046 & 0.4099 & $0.07 I$ & 0.013 & 0.1641 \\
\hline Left & 0.098 & 0.035 & & 0.027 & 0.014 & & 0.155 & 0.05 & & 0.067 & 0.015 & \\
\hline
\end{tabular}


Table 3 Correlation Between Nerve Size and BMI and Age. PCC: Pearson Correlation Coefficient

\begin{tabular}{|l|c|c|c|c|c|c|c|c|}
\hline \multirow{2}{*}{$\begin{array}{l}\text { Nerve Size } \\
\text { Average) }\end{array}$} & \multicolumn{4}{|c|}{ BMI } & \multicolumn{4}{c|}{ Age } \\
\cline { 2 - 9 } & $\mathbf{2 0 - 2 4}$ & $\mathbf{2 5 - 3 0}$ & $\mathbf{3 0 +}$ & Overall PCC & $\mathbf{2 0 - 2 9}$ & $\mathbf{3 0 - 3 9}$ & $\mathbf{4 0 +}$ & Overall PCC \\
\hline Median & 0.090 & 0.105 & 0.085 & 0.064 & 0.087 & 0.104 & 0.104 & 0.169 \\
Musculocutaneous & 0.025 & 0.027 & 0.032 & 0.305 & 0.028 & 0.027 & 0.026 & -0.034 \\
Radial & 0.129 & 0.164 & 0.181 & 0.485 & 0.128 & 0.153 & 0.179 & 0.445 \\
Ulnar & 0.064 & 0.07 & 0.076 & 0.347 & 0.064 & 0.073 & 0.07 & 0.090 \\
\hline
\end{tabular}

musculocutaneous, and radial nerves between each gender. When comparing the position of the nerves on each side of the body, the radial nerve was found to be most commonly in different places on the right and left. In general, there is also clear evidence of distinct overlap between the positions of each of the different nerves of the brachial plexus.

Variations in the anatomy of the brachial plexus have been well documented in literature since the early 1900s, and yet very little research has been done regarding contributing factors to these differences. ${ }^{6-8}$ Uysal et al found that of the brachial plexuses of 200 foetuses, there were variations present in $53.5 \%$ of the brachial plexuses examined. ${ }^{8}$ Pandey et al reported variations in the position of the nerves in $12.8 \%$ in their dissection of 172 cadavers. $^{7}$ Similarly, in an ultrasound study of 69 patients, variations were found in $59 \%$ of the participants. ${ }^{9}$ These variations could impact upon the effectiveness of brachial plexus blocks in patients. ${ }^{11,12}$
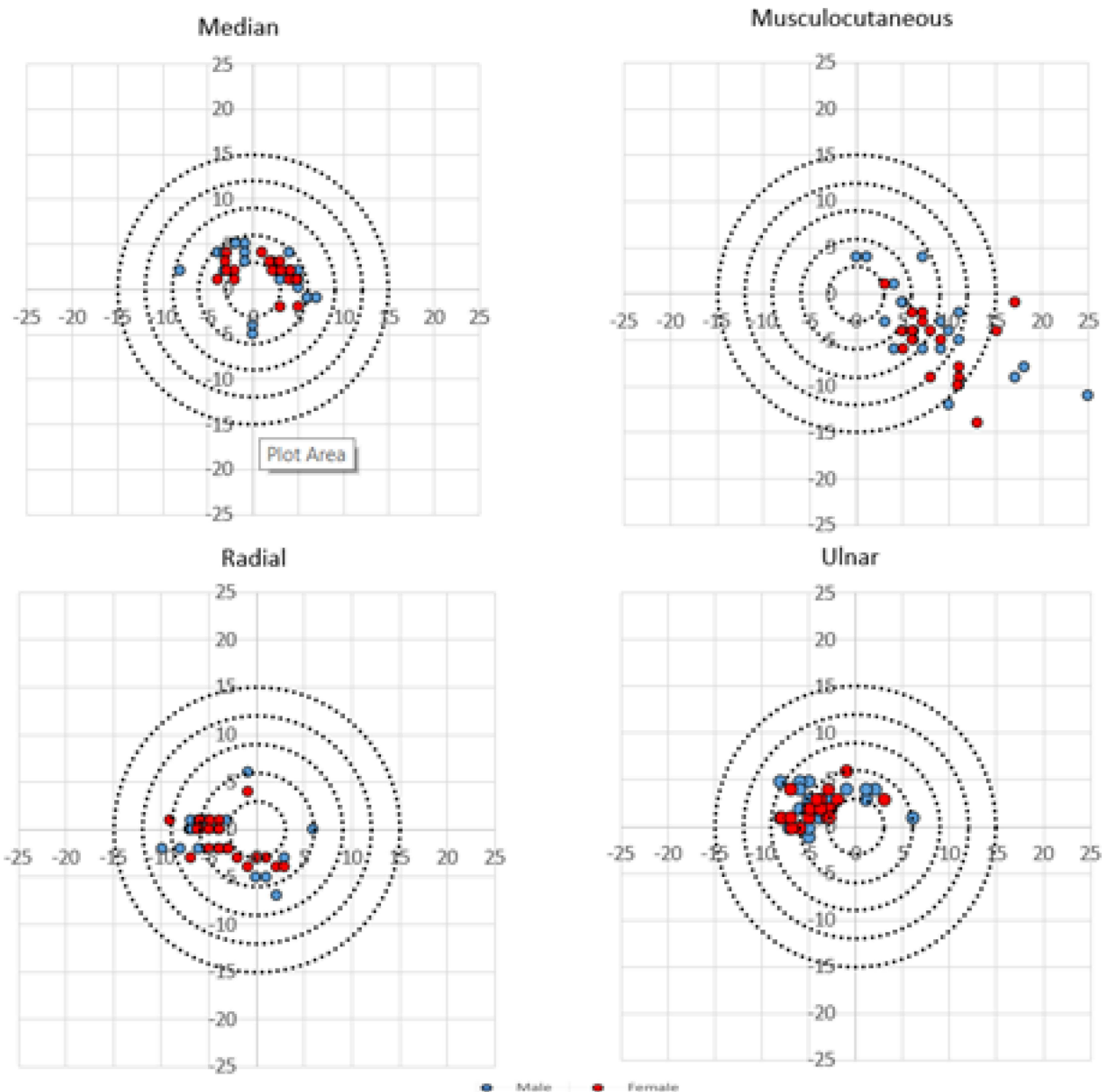

Figure $\mathbf{2}$ The position of each of the nerves of the brachial plexus in males and females. 

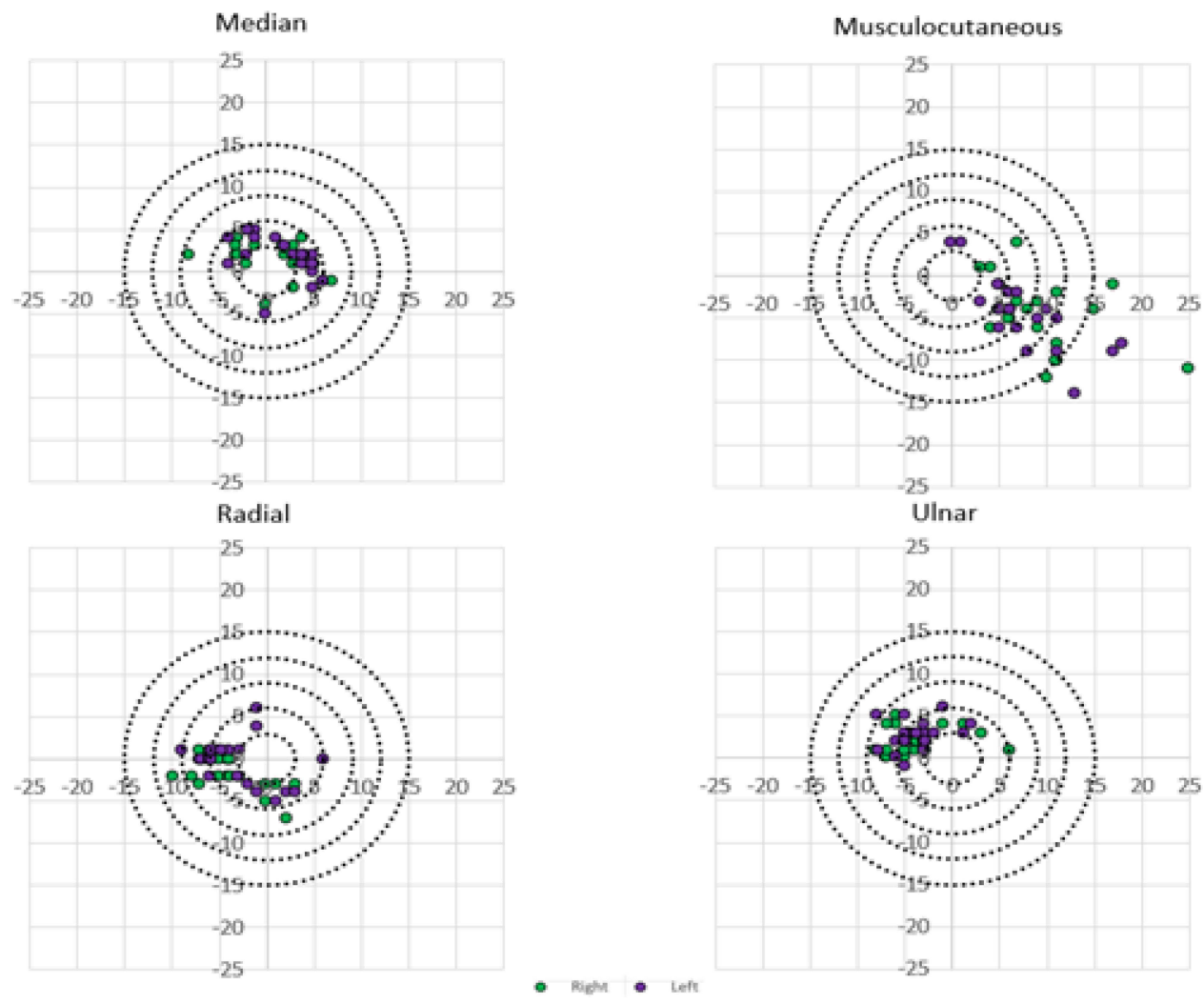

Figure 3 The position of each of the nerves of the brachial plexus on the right and left hand side.

A thorough understanding of the anatomy of the brachial plexus is key to successful nerve blockade anaesthesia during upper limb surgeries. The anatomical anomalies found in this study could impact on the effectiveness and accuracy of anaesthesia and hence it is important to have an understanding of any variations that occur and in turn anything that may be causing these variations. These findings further emphasise the value of ultrasound in performance of the axillary brachial plexus block.

Our significant findings from this study relate mostly to differences between genders. Although correlations were seen between the radial, musculocutaneous and ulnar nerve and BMI and between the radial nerve and age, the study was not sufficiently powered to detect statistical significance. The weak positive correlation between age and size of nerves may explain the reduced minimum effective volume for ultrasound-guided brachial plexus block in elderly patients. ${ }^{13}$

Our results showed that the mean size of all nerves was bigger in males than females, although this result was only significant for the musculocutaneous, radial and ulnar nerves. The anaesthetic implications of this finding relate to the effectiveness of brachial blocks. A larger nerve may require a greater dose of anaesthetic agent; ${ }^{14}$ however, the correlation between the size of the nerve and BMI was very small which has been reflected in clinical dose-finding studies. ${ }^{15}$ This is an area that could be studied further, specifically looking at height and nerve size, rather than weight, as height is a given constant but that weight is affected by lifestyle.

Another factor not considered in this study but which may impact on the size of nerves is the dominant side of an individual. There is an argument for the nerves on the dominant side of an individual to be larger due to more regular use. This is another possible focus area for a future study. The difference in the demographics of the patients may help explain the wide variation in the effective doses seen between different studies using similar techniques and agents. ${ }^{16,17}$

\section{Limitations}

This study had a number of limitations. First, the study had a relatively small sample size of only 20 participants. Due to this, this study can only act as a pilot for future studies with a larger sample size. In addition, the participants for the study were all taken from the staff at the Hand Unit of Leeds General Infirmary so they may not be representative of the general population. With the use of ultrasound, there is potential that 
the image taken from each participant may not be in the exact same position, meaning that each image could be showing slightly different areas of the brachial plexus leading to a less accurate comparison. Secondly, when plotting the position of the nerves onto the graph, the center point of each nerve was used. However, due to the irregular shape of the nerves for some of the images it was hard to determine the centre of the nerve. This could result in the graphs inaccurately representing the position of the nerves in the brachial plexus.

\section{Conclusion}

This is the first study that has examined the effect of gender, age, BMI and the side of the body on the inter-individual and intra-individual size and position of the nerves of the brachial plexus in the axilla. The results have shown that the median, musculocutaneous and radial nerves tend to be larger in males than in females. In addition, it was found that the position of the nerves of the brachial plexus can vary between genders, side of body and also overlap each other. However, the sample size limits the significance of the results of the study.

This study acts as a pilot study for a further larger study into the effects of these factors on the size and the position of the nerves of the brachial plexus.

\section{Ethics}

This study was conducted in accordance with the Declaration of Helsinki.

\section{Acknowledgments}

Dr Andew Berrill and Dr Charlotte Hurren for providing ultrasound images and data collection.

\section{Funding}

There is no funding to report.

\section{Disclosure}

The authors declare no conflicts of interest.

\section{References}

1. Smith T, Pinnock C, Lin T. Fundamentals of Anaesthesia. Cambridge University Press; 2009.

2. Standring S. Gray's anatomy E-Book: the anatomical basis of clinical practice. Elsevier Health Sciences; 2015.

3. Neal JM, Gerancher J, Hebl JR, et al. Upper extremity regional anesthesia: essentials of our current understanding, 2008. Reg Anesth Pain Med. 2009;34(2):134.

4. Gulur P, Nishimori M, Ballantyne JC. Regional anaesthesia versus general anaesthesia, morbidity and mortality. Best Pract Res Clin Anaesthesiol. 2006;20(2):249-263. doi:10.1016/j.bpa.2005.10.002

5. Hopkins PM. Ultrasound guidance as a gold standard in regional anaesthesia. Br J Anaesth. 2007;98(3):299-301. doi:10.1093/bja/ael387

6. Kerr AT. The brachial plexus of nerves in man, the variations in its formation and branches. Dev Dyn. 1918;23(2):285-395.

7. Pandey S, Shukla V. Anatomical variations of the cords of brachial plexus and the median nerve. Clin Anat. 2007;20(2):150-156. doi:10.1002/ca.20365

8. Uysal II, Şeker M, Karabulut AK, Büyükmumcu M, Ziylan T. Brachial plexus variations in human fetuses. Neurosurgery. 2003;53 (3):676-684. doi:10.1227/01.NEU.0000079485.24016.70

9. Retzl G, Kapral S, Greher M, Mauritz W. Ultrasonographic findings of the axillary part of the brachial plexus. Anesth Analg. 2001;92 (5):1271-1275. doi:10.1097/00000539-200105000-00037

10. Rueden CT, Schindelin J, Hiner MC, et al. ImageJ2: imageJ for the next generation of scientific image data. BMC Bioinform. 2017;18 (1):529. doi:10.1186/s12859-017-1934-z

11. Nowrouz MY, Huntoon MA. Precise anatomical targeting: location, Location, Location! Reg Anesth Pain Med. 2017;42(5):554-555. doi:10.1097/AAP.0000000000000651

12. Denny NM, Harrop-Griffiths W. Location, location, location! Ultrasound imaging in regional anaesthesia. Br J Anaesth. 2005;94 (1):1-3. doi:10.1093/bja/aei001

13. Pavicic Saric J, Vidjak V, Tomulic K, Zenko J. Effects of age on minimum effective volume of local anesthetic for ultrasound-guided supraclavicular brachial plexus block. Acta Anaesthesiol Scand. 2013;57(6):761-766. doi:10.1111/aas.12109

14. Eichenberger U, Stockli S, Marhofer P, et al. Minimal local anesthetic volume for peripheral nerve block: a new ultrasound-guided, nerve dimension-based method. Reg Anesth Pain Med. 2009;34 (3):242-246. doi:10.1097/AAP.0b013e31819a7225

15. Gupta PK, Pace NL, Hopkins PM. Effect of body mass index on the ED50 volume of bupivacaine $0.5 \%$ for supraclavicular brachial plexus block. Br J Anaesth. 2010;104(4):490-495. doi:10.1093/bja/ aeq017

16. Duggan E, El Beheiry H, Perlas A, et al. Minimum effective volume of local anesthetic for ultrasound-guided supraclavicular brachial plexus block. Reg Anesth Pain Med. 2009;34(3):215-218. doi:10.1097/AAP.0b013e31819a9542

17. Gupta PK, Hopkins PM. Effect of concentration of local anaesthetic solution on the ED (5)(0) of bupivacaine for supraclavicular brachial plexus block. Br J Anaesth. 2013;111(2):293-296. doi:10.1093/bja/ aet033
Local and Regional Anesthesia

\section{Publish your work in this journal}

Local and Regional Anesthesia is an international, peer-reviewed, open access journal publishing on the development, pharmacology, delivery and targeting and clinical use of local and regional anesthetics and analgesics. The journal welcomes submitted papers covering original research, basic science, clinical studies, reviews \& evaluations, guidelines, expert opinion and commentary, case reports and extended reports. The manuscript management system is completely online and includes a very quick and fair peer-review system, which is all easy to use. Visit http://www.dovepress.com/testimonials. php to read real quotes from published authors. 